\title{
Atypical Omenn Syndrome due to Adenosine Deaminase Deficiency
}

\author{
Avni Y. Joshi, ${ }^{1,2}$ Erin K. Ham, ${ }^{2}$ Neel B. Shah, ${ }^{3}$ Xiangyang Dong, ${ }^{4}$ \\ Shakila P. Khan, ${ }^{5}$ and Roshini S. Abraham ${ }^{4}$ \\ ${ }^{1}$ Division of Pediatric and Adult Allergy and Immunology, Department of Pediatric and Adolescent Medicine, \\ Mayo Clinic, Rochester, MN 55905, USA \\ ${ }^{2}$ Department of Internal Medicine, Mayo Clinic, Rochester, MN 55905, USA \\ ${ }^{3}$ Department of Medical Genetics, Mayo Clinic, Rochester, MN 55905, USA \\ ${ }^{4}$ Department of Laboratory Medicine and Pathology, Mayo Clinic, Rochester, MN 55905, USA \\ ${ }^{5}$ Division of Pediatric Hematology and Oncology, Department of Pediatric and Adolescent Medicine, Mayo Clinic, \\ Rochester, MN 55905, USA
}

Correspondence should be addressed to Avni Y. Joshi, joshi.avni@mayo.edu

Received 14 February 2012; Accepted 8 April 2012

Academic Editors: I. A. Hagel and A. Plebani

Copyright ( $) 2012$ Avni Y. Joshi et al. This is an open access article distributed under the Creative Commons Attribution License, which permits unrestricted use, distribution, and reproduction in any medium, provided the original work is properly cited.

We present here a novel case of an atypical Omenn syndrome (OS) phenotype due to mutations in the ADA gene encoding adenosine deaminase. This case is noteworthy for a significant increase in circulating CD56 ${ }^{\text {bright }}$ CD16- cytokine-producing NK cells after treatment with steroids for skin rash.

\section{Introduction}

Omenn syndrome (OS) is a distinct manifestation of Severe Combined Immunodeficiency (SCID) characterized by erythroderma, hepatosplenomegaly, lymphadenopathy, eosinophilia, and elevated IgE and alopecia [1-3]. Patients with OS usually present in infancy with viral or fungal pneumonitis, chronic diarrhea, and failure to thrive, but in contrast to classic SCID patients also have the abovementioned characteristics.

Here we present a case of atypical OS in an infant with adenosine deaminase (ADA) deficiency.

\section{Case Report}

A 2-month-old Somali boy, born to consanguineous parents, presented to our hospital with concerns for immunodeficiency. The initial symptoms included cough, diffuse skin rash (Figure 1), hepatosplenomegaly, and a sepsislike syndrome. A complete sepsis workup was negative, except for an absolute lymphocyte count (ALC) of 0 , which triggered evaluation for an underlying primary immunodeficiency. T-, B- and NK-cell quantitation in blood revealed a TB-NK- phenotype consistent with Severe Combined Immunodeficiency (SCID). The molecular defect was likely thought to be in the $A D A$ gene due to the ethnicity of the patient and the lymphocyte phenotype, therefore, ADA levels were tested and found to be 0 (reference range: $0.3-1.5 / \mathrm{g} \mathrm{Hb}$ ).

Broad-spectrum antibiotics were initiated, but with negative cultures, were maintained on prophylactic antibiotics (Bactrim for Pneumocystis, initial Fluconazole followed by Caspofungin for antifungal, IVIG and Palivizumab for RSV).

His skin rash was extensively evaluated and the pathology was initially regarded as possible graft versus host disease due to maternal engraftment [4], due to the presence of apoptotic keratinocytes. However, the absence of circulating $\mathrm{T}$ cells argued against that possibility and chimerism [5] studies on a skin biopsy revealed all cells to be XY in origin, confirming absence of maternal engraftment. Treatment was initiated with steroids and PEG-ADA (biweekly, $60 \mathrm{U} / \mathrm{kg} /$ week due to the absence of a matched donor for stem cell transplantation and unsuitability at the time for 


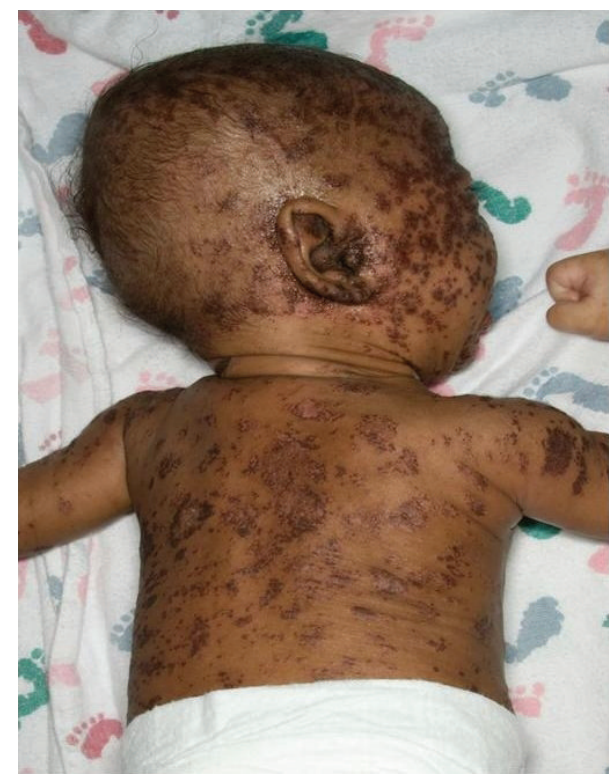

FIGURE 1: Diffuse rash in an infant with ADA deficiency.

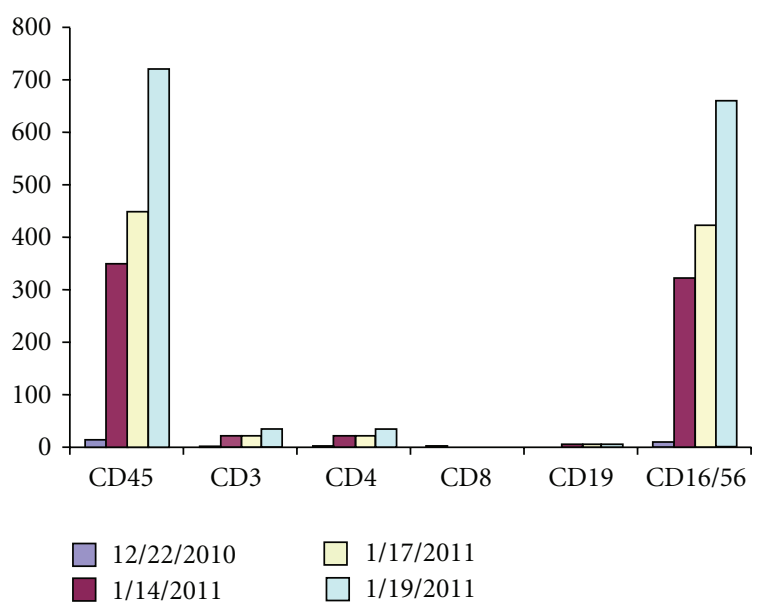

Figure 2: Serial T, B, and NK lymphocyte measurements.

gene therapy [6]; trough plasma ADA levels were monitored while on PEG-ADA treatment-range of $30-40 \mathrm{mmol} / \mathrm{hr} / \mathrm{mL}$ (target levels > $12 \mathrm{mmol} / \mathrm{hr} / \mathrm{mL}$ ), but shortly thereafter, there was a remarkable increase in circulating lymphocyte counts with doubling in a span of 5 days. Lymphocyte immunophenotyping revealed these to be primarily NK cells (CD16/56), with a small number of T cells (Figure 2, PEG ADA started on 01/03/2011).

Analysis of thymic function (CD4 recent thymic emigrants: CD4+ CD45RA+ CD31+) revealed an almost complete lack of naive $\mathrm{T}$ cells, contrary to what would typically be expected for age, and $\mathrm{T}$ cells that were present had the memory, CD45RO+ phenotype, as often seen in OS. Flow cytometric analysis of the NK-cell population revealed an unusual expansion of cytokine-producing NK cells, CD56 ${ }^{\text {bright }} \mathrm{CD} 16-$, which is typically only $10 \%$ of circulating NK cells (Figure 3). The appearance of this unusually expanded NK cell subset and small numbers of CD45RO+CD4+ $\mathrm{T}$ cells in blood was likely related to retrafficking of these cells from the skin lesions rather than de novo generation due to ADA treatment due to the short time-interval posttreatment in which the cells were detected. There has previously been one report of skin-infiltrating CD56 ${ }^{\text {bright }} \mathrm{CD} 16-\mathrm{NK}$ cells in a patient with X-linked (IL2RG mutation) SCID who had OS-like features [7].

The patient reported herein had several characteristics of OS as mentioned but was atypical for the presence of the expanded CD56 ${ }^{\text {br }}$ CD16- NK cells, normal IgE levels, and lack of eosinophilia.

Molecular analysis of the $A D A$ gene in both parents revealed a heterozygous $\mathrm{Q} 3 \mathrm{X}$ nonsense mutation (c. $7 \mathrm{C}>\mathrm{T}$; CAG $>$ TAG), which has been previously reported to be 


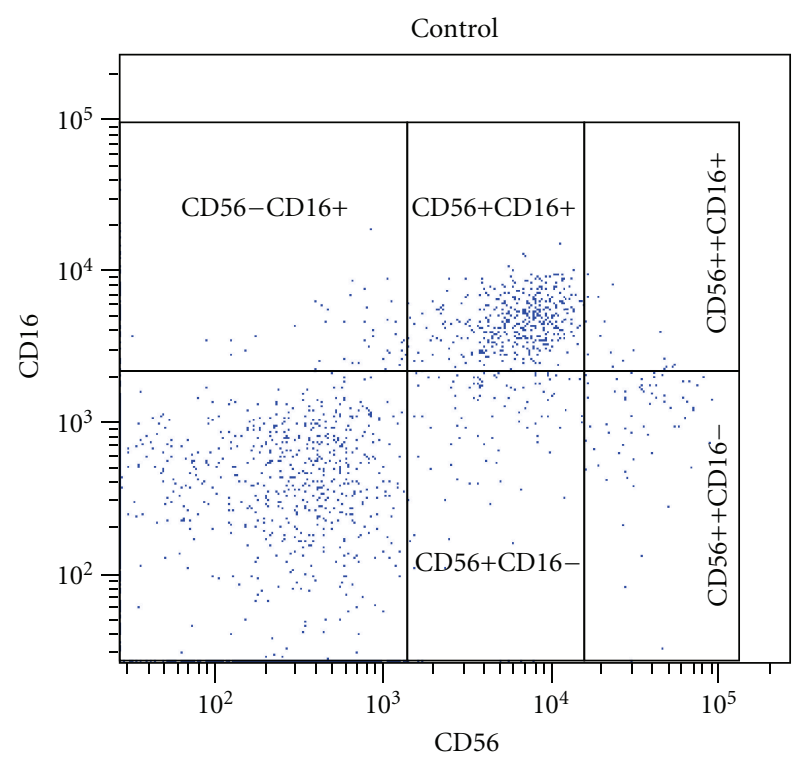

(a)

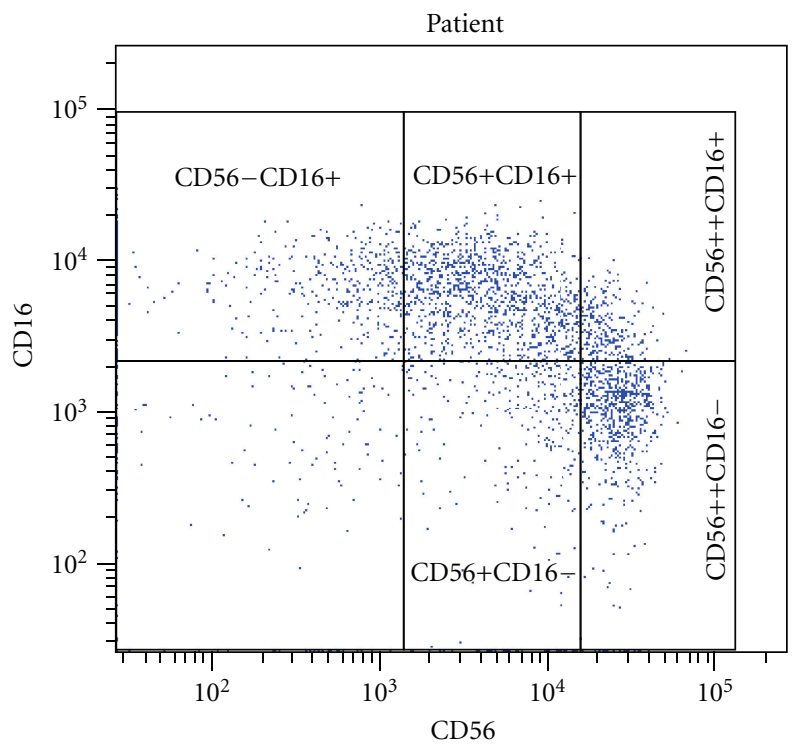

(b)

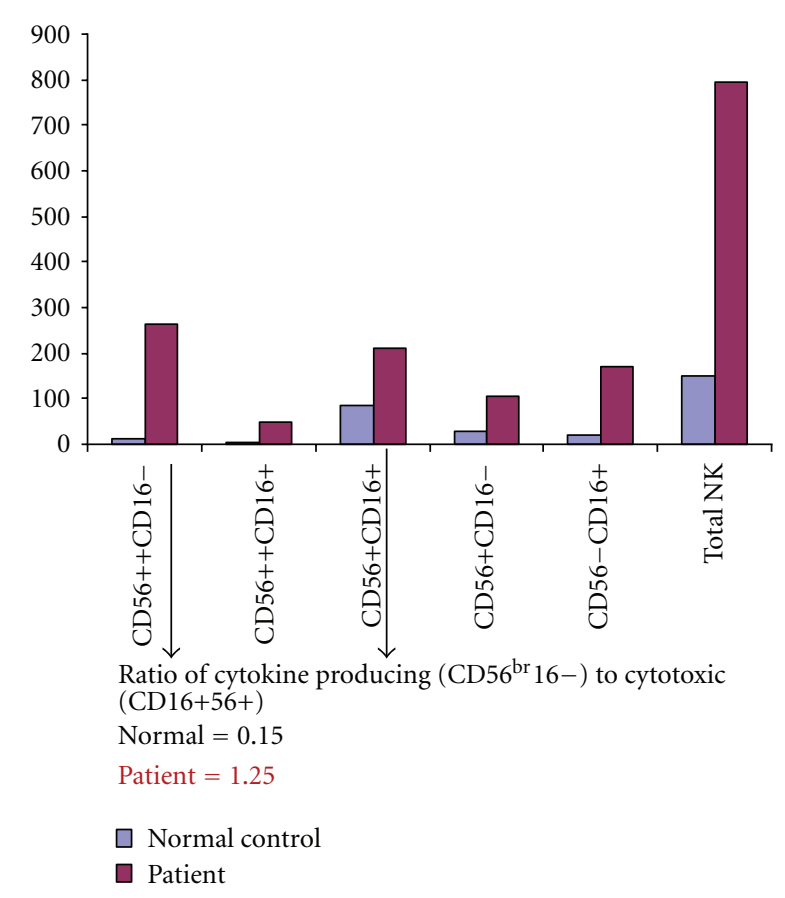

(c)

FIgURE 3: Natural Killer (NK) cell phenotyping.

present at a frequency of at least 1 in 5000 to 1 in 10,000 in the Somali population [8].

Despite use of high-dose steroids, his respiratory status worsened and he developed seizures. A spinal tap showed evidence of human herpes virus-6 (HHV 6) via PCR with HHV 6 viremia Bronchial alveolar lavage that revealed Stenotrophomonas maltophilia and HHV6. Treatment was initiated with Foscarnet alone followed by combination ganciclovir. Despite aggressive intervention, he continued to be ventilator dependent with worsening pulmonary hypertension due to chronic lung disease. Eventually, the decision to cease supportive care was made in conjunction with the family 40 days after hospitalization.

This is the first report, to the best of our knowledge, of an atypical Omenn syndrome due to ADA deficiency with expansion of CD56 ${ }^{\text {br }} \mathrm{CD} 16-\mathrm{NK}$ cells. Only 2 other patients with ADA-SCID and features of Omenn syndrome have been reported $[9,10]$. The variability in the phenotypic spectrum 
of classic SCID-associated genes emphasizes the necessity of genotype-phenotype correlations.

\section{Acknowledgment}

The authors would like to thank Dr. Michael Hershfield, MD, Duke University Medical Center for his kind assistance in evaluating ADA levels pre- and posttreatment.

\section{References}

[1] G. S. Omenin, "Familial reticuloendotheliosis with eosinophilia," The New England journal of medicine, vol. 273, pp. 427-432, 1965.

[2] M. Kato, H. Kimura, M. Seki et al., "Omenn syndromereview of several phenotypes of Omenn syndrome and RAG1/ RAG2 mutations in Japan," Allergology international, vol. 55, no. 2, pp. 115-119, 2006.

[3] A. Villa, L. D. Notarangelo, and C. M. Roifman, "Omenn syndrome: inflammation in leaky severe combined immunodeficiency," Journal of Allergy and Clinical Immunology, vol. 122, no. 6, pp. 1082-1086, 2008.

[4] A. Sottini, E. Quiros-Roldan, L. D. Notarangelo, A. Malagoli, D. Primi, and L. Imberti, "Engrafted maternal T cells in a severe combined immunodeficiency patient express T-cell receptor variable beta segments characterized by a restricted V-D-J junctional diversity," Blood, vol. 85, no. 8, pp. 21052113, 1995.

[5] A. J. Sieverkropp, R. G. A. Andrews, L. Gaur, and L. E. Shields, "Chimerism analysis by sex determining region $\mathrm{Y}$ (SRY) and major histocompatibility complex markers in non-human primates using quantitative real-time polymerase chain reaction," Tissue Antigens, vol. 66, no. 1, pp. 19-25, 2005.

[6] M. S. Hershfield, S. Chaffee, R. U. Sorensen, R. Hirschhorn, and E. W. Gelfand, "Enzyme replacement therapy with polyethylene glycol-adenosine deaminase in adenosine deaminase deficiency: overview and case reports of three patients, including two now receiving gene therapy," Pediatric Research, vol. 33, no. 1, pp. S42-S48, 1993.

[7] F. Shibata, T. Toma, T. Wada et al., "Skin infiltration of CD56bright CD16- natural killer cells in a case of X-SCID with Omenn syndrome-like manifestations," European Journal of Haematology, vol. 79, no. 1, pp. 81-85, 2007.

[8] J. J. Sanchez, G. Monaghan, C. Børsting, G. Norbury, N. Morling, and H. B. Gaspar, "Carrier frequency of a nonsense mutation in the Adenosine Deaminase (ADA) gene implies a high incidence of ADA-deficient Severe combined immunodeficiency (SCID) in Somalia and a single, common haplotype indicates common ancestry," Annals of Human Genetics, vol. 71, no. 3, pp. 336-347, 2007.

[9] I. Dalal, D. Tasher, R. Somech et al., "Novel mutations in RAG1/2 and ADA genes in Israeli patients presenting with TB- SCID or Omenn syndrome," Clinical Immunology, 2011.

[10] C. M. Roifman, J. Zhang, A. Atkinson, E. Grunebaum, and K. Mandel, "Adenosine deaminase deficiency can present with features of Omenn syndrome," Journal of Allergy and Clinical Immunology, vol. 121, no. 4, pp. 1056-1058, 2008. 


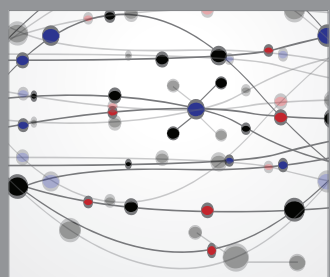

The Scientific World Journal
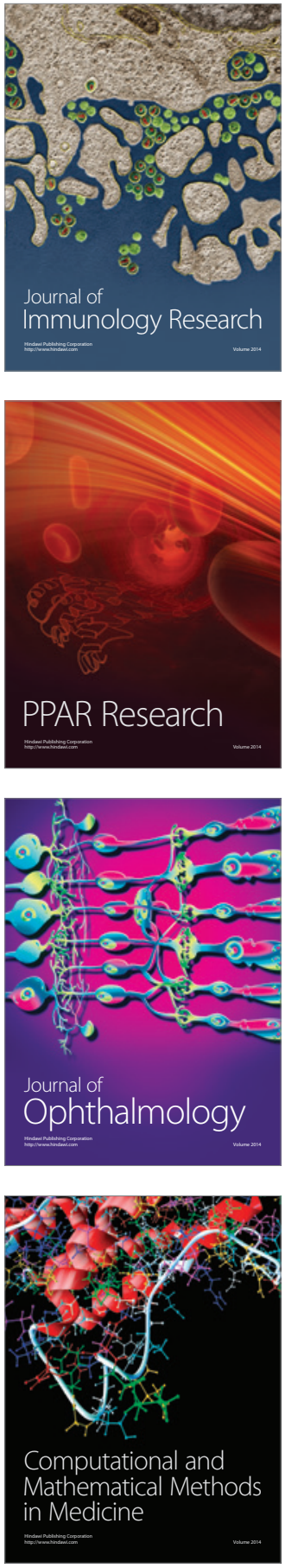

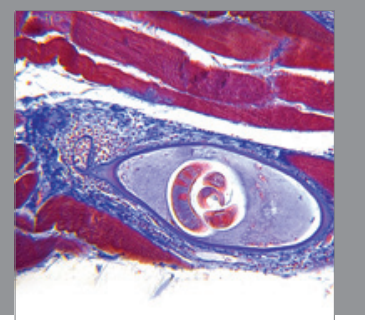

Gastroenterology

Research and Practice
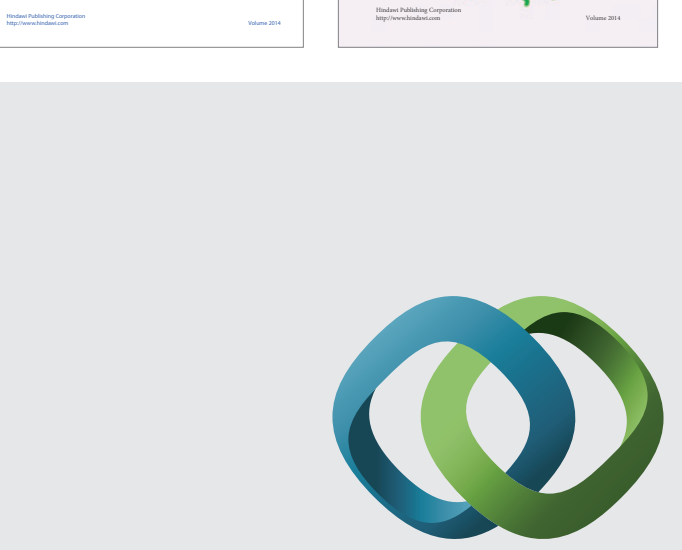

\section{Hindawi}

Submit your manuscripts at

http://www.hindawi.com
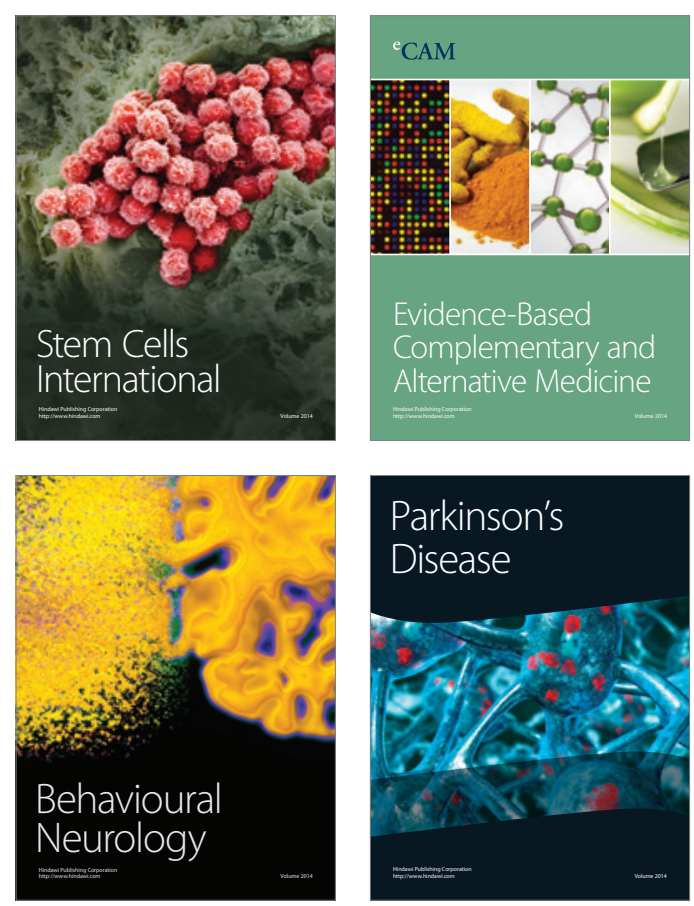

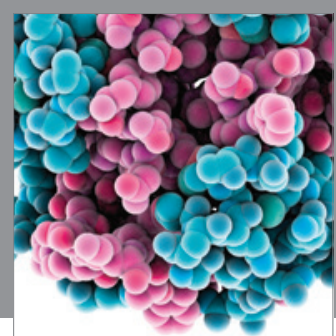

Journal of
Diabetes Research

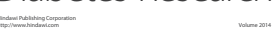

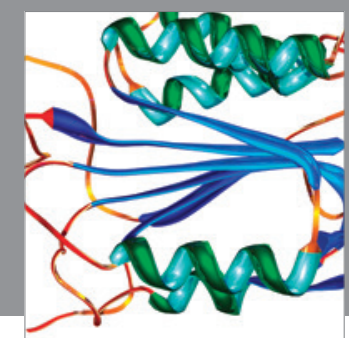

Disease Markers
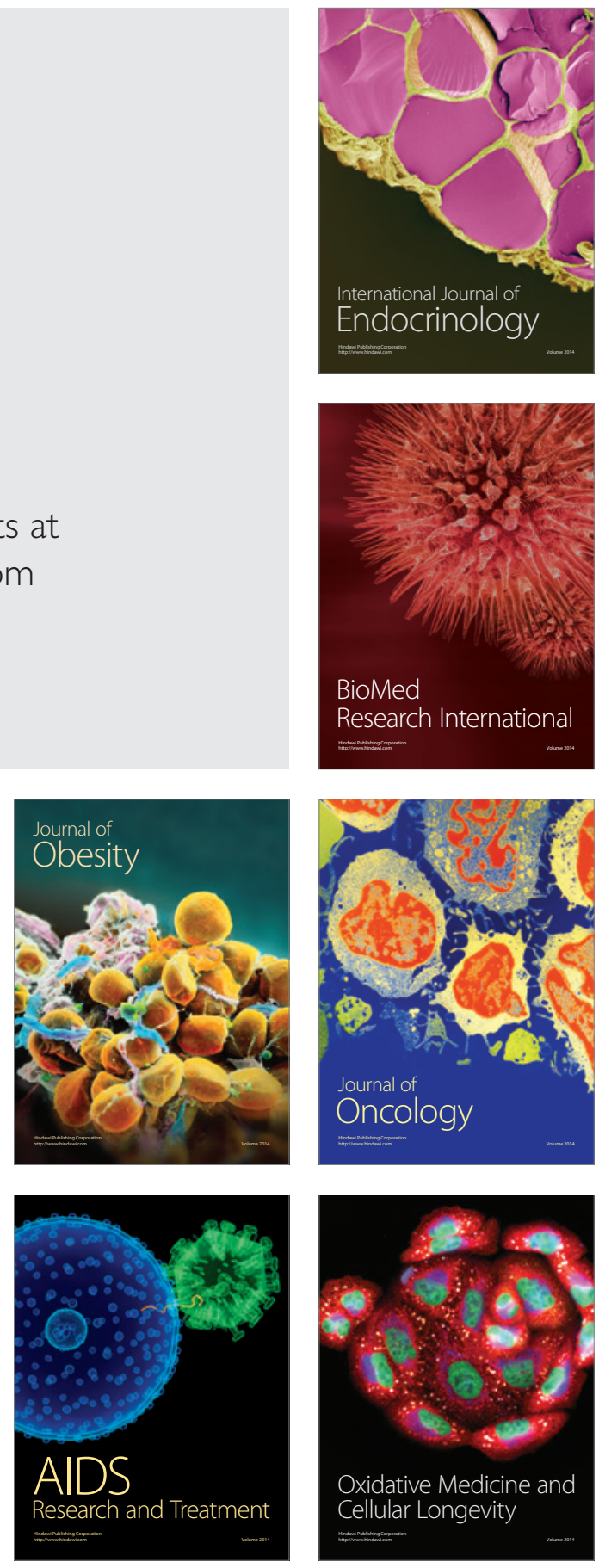